\title{
Nintedanib: A Review in Fibrotic Interstitial Lung Diseases
}

\author{
Yvette N. Lamb ${ }^{1}$
}

Accepted: 23 February 2021

(c) Springer Nature 2021, corrected publication 2021

\begin{abstract}
Progressive fibrosing interstitial lung diseases (ILDs) involve similar pathophysiological processes, indicating the potential for common approaches to treatment. Nintedanib $\left(\mathrm{Ofev}^{\circledR}\right)$, an intracellular tyrosine kinase inhibitor (TKI) with antifibrotic properties, was one of the first drugs approved for use in idiopathic pulmonary fibrosis (IPF) and has more recently been approved for use in other chronic fibrosing ILDs with a progressive phenotype and systemic sclerosis-associated ILD (SScILD). In multinational phase III trials, nintedanib significantly reduced the annual rate of decline in forced vital capacity (FVC) in adults with IPF, other progressive fibrosing ILDs and SSc-ILD. Reductions in FVC decline with nintedanib in patients with IPF and severe gas exchange impairment were comparable to those in patients with milder disease. Real-world experience in patients with IPF supports the effectiveness of nintedanib in slowing ILD progression. Nintedanib had a manageable tolerability profile in patients with fibrotic ILDs in clinical trials and real-world studies. No new safety signals have emerged from global pharmacovigilance data. Nintedanib continues to represent an important therapeutic option in patients with IPF and is the first drug to be approved for use in patients with other chronic fibrosing ILDs with a progressive phenotype or SSc-ILD, with these approvals expanding the range of fibrotic ILDs for which nintedanib can be prescribed.
\end{abstract}

\section{Plain language summary}

Treatment options for fibrotic interstitial lung diseases (ILDs) that involve progressive lung function decline have historically been limited. Nintedanib $\left(\mathrm{Ofev}^{\circledR}\right)$ was one of the first antifibrotic drugs to be approved for use in idiopathic pulmonary fibrosis and is now also approved for use in other chronic fibrosing ILDs with a progressive phenotype and systemic sclerosisassociated ILD (SSc-ILD). Nintedanib reduced lung function deterioration in patients with idiopathic pulmonary fibrosis, other chronic fibrosing ILDs with a progressive phenotype and SSc-ILD in well-designed clinical trials. In patients with idiopathic pulmonary fibrosis, the clinical benefit of nintedanib was shown to persist over more than 4 years of treatment. The most common adverse events in nintedanib recipients were diarrhoea and nausea, which were manageable in the majority of patients. Real-world experience supports the effectiveness and acceptable safety of nintedanib. Nintedanib remains an important treatment option for patients with idiopathic pulmonary fibrosis and is the first drug to be approved for use in patients with other chronic fibrosing ILDs with a progressive phenotype and SSc-ILD.

Digital Features for this Adis Drug Evaluation can be found at https://doi.org/10.6084/m9.figshare.13654466.

The manuscript was reviewed by: $\boldsymbol{S}$. A. Antoniu, Department of Medicine II - Nursing/Palliative Care, Grigore T. Popa University of Medicine and Pharmacy, Iaşi, Romania; $\boldsymbol{P}$. $\boldsymbol{S}$. Burge, Occupational Lung Disease Unit, Birmingham Heartlands Hospital, Birmingham, UK; A. U. Wells, Interstitial Lung Disease Unit, Royal Brompton Hospital, London, UK.

Yvette N. Lamb

demail@springer.com

1 Springer Nature, Private Bag 65901, Mairangi Bay, Auckland 0754, New Zealand

\section{Introduction}

Idiopathic pulmonary fibrosis (IPF) is a progressive fibrosing interstitial lung disease (ILD) and, as such, is characterized by self-sustaining fibrosis, deteriorating lung function, worsening symptoms (e.g. dyspnoea, cough), increasingly impaired healthrelated quality of life (HR-QOL) and premature mortality [1-3]; the median survival from the time of diagnosis is 4.5 years [4]. Other ILDs that may develop a progressive fibrosing phenotype include (among others) idiopathic non-specific interstitial pneumonia, unclassifiable idiopathic interstitial pneumonia, hypersensitivity pneumonitis, and autoimmune ILDs such as rheumatoid arthritis-associated ILD and systemic sclerosis-associated ILD 
Nintedanib: clinical considerations in fibrotic ILDs

Orally administered TKI with antifibrotic properties

Reduces lung function deterioration in patients with IPF, other chronic fibrosing ILDs with a progressive phenotype and SSc-ILD

Clinical benefit persists over long-term treatment

Manageable tolerability profile; most common adverse events are gastrointestinal

(SSc-ILD) $[1,5,6]$. These progressive fibrosing ILDs can have similar clinical characteristics to IPF (although unlike IPF, they may be clinically stable, particularly in cases of milder disease) and appear to have genetic risk factors and pathophysiological mechanisms that overlap with those of IPF, suggesting the potential for common treatment approaches $[1,5,6]$.

Early treatment of IPF is crucial for slowing lung function decline, reducing the risk of acute exacerbations and improving clinical outcomes [3]. Treatment options for IPF have historically been limited, however, and until recently there were no approved drugs for the treatment of non-IPF ILDs [2]. The fibroblast growth factor (FGF), platelet-derived growth factor (PDGF) and vascular endothelial growth factor (VEGF) receptor tyrosine kinases have proved to be valuable therapeutic targets in the treatment of IPF, with FGF, PDGF and VEGF mediating profibrotic processes and being implicated in IPF pathogenesis [7].

Nintedanib $\left(\mathrm{Ofev}^{\circledR}\right)$, an orally administered intracellular tyrosine kinase inhibitor (TKI) initially developed as an antitumor agent, has antifibrotic properties and was one of the first drugs (along with the antifibrotic pirfenidone) to be approved for the treatment of IPF in the EU and USA [8]; use of nintedanib in this indication has been previously reviewed in Drugs [7]. More recently, nintedanib has been approved in the same regions for use in other chronic fibrosing ILDs with a progressive phenotype and SSc-ILD (Sect. 4). This article discusses the therapeutic efficacy and safety/tolerability of nintedanib in the treatment of fibrotic ILDs. A brief overview of the pharmacological properties of nintedanib relevant to its use in fibrotic ILDs is presented in Table 1.

\section{Therapeutic Efficacy of Nintedanib}

\subsection{In IPF}

The efficacy of nintedanib in the treatment of patients with IPF was investigated in the randomized, double-blind, placebo-controlled, multinational, phase III INPULSIS-1 and INPULSIS-2 trials [9] and an open-label long-term extension (INPULSISON) [10]. The dosage evaluated in the INPULSIS trials (nintedanib $150 \mathrm{mg}$ twice daily) was established in an earlier phase II dose-finding trial (TOMORROW) [11]; given that phase III data are available, TOMORROW is not discussed further. Data from the INPULSIS trials are supported by those from the randomized, double-blind, multinational, phase III INSTAGE trial evaluating nintedanib plus sildenafil versus nintedanib alone in the treatment of patients with advanced IPF [12] and numerous real-world studies (e.g. [13-20]).

\subsubsection{INPULSIS-1 and INPULSIS-2}

The replicate INPULSIS- 1 and INPULSIS- 2 trials enrolled adults $\geq 40$ years of age diagnosed with IPF within the previous 5 years [9]. Eligibility was confirmed by means of centrally reviewed high-resolution computed tomography (HRCT) of the chest (conducted within the previous 12 months) and, where available, lung biopsy specimens. Patients were required to have a forced vital capacity (FVC) $\geq 50 \%$ of the predicted value and a carbon monoxide diffusion capacity ( $\mathrm{DL}_{\mathrm{CO}}$; corrected for haemoglobin) 30-79\% of the predicted value. Background therapy with prednisone $\leq 15 \mathrm{mg}$ /day (or equivalent) was permitted if the dosage had been stable for $\geq 8$ weeks prior to screening [9].

Patients received oral nintedanib $150 \mathrm{mg}$ twice daily or matching placebo for 52 weeks [9]. Dose interruption or reduction to nintedanib $100 \mathrm{mg}$ twice daily was permitted for the management of adverse events (AEs). In both trials, the primary endpoint was the annual rate of decline in FVC over 52 weeks. Baseline characteristics of patients were similar between treatment groups in each trial. At baseline, patients had a mean age $\approx 67$ years, FVC $\approx 80 \%$ of the predicted value and $\mathrm{DL}_{\mathrm{CO}} \approx 47 \%$ of the predicted value. In each trial, the mean exposure to the study drug was $\approx 45$ weeks [9].

Nintedanib was effective in reducing FVC decline [9]. Annual rates of FVC decline were significantly lower in nintedanib recipients than placebo recipients in INPULSIS-1, INPULSIS-2 and a prespecified pooled analysis (Table 2). Nintedanib also significantly reduced the adjusted absolute mean change from baseline in FVC in $\mathrm{mL}$ and as a percentage of the predicted value ( $p<0.001$ vs placebo in each trial). At week 52 , significantly ( $p \leq 0.001$ ) higher proportions of nintedanib than placebo recipients did not experience an absolute decline in the percentage of predicted FVC of more than 5\% (both trials) or of more than $10 \%$ (INPULSIS-1 only) [9]; in the pooled dataset, significantly $(p<0.001)$ more nintedanib than placebo recipients did not experience an FVC decline of more than 5\% (53\% vs 39\%) or of more than $10 \%$ (70\% vs $61 \%$ ) [9] and $25 \%$ of nintedanib recipients versus $9 \%$ of placebo recipients had either an improvement or no decline in FVC based on annual rate of FVC change [21]. 


\section{Pharmacodynamic properties}

Small molecule tyrosine kinase inhibitor that competitively binds to ATP binding pocket of kinases to block intracellular signalling cascades [37, 51, 70]; potently inhibits receptor tyrosine kinases implicated in pathogenesis of ILDs, including VEGFR-1, -2 and -3 (IC $_{50}$ values 13-34 nmol/L), FGFR-1, -2 and -3 (37-108 nmol/L), PDGFR- $\alpha$ (59 nmol/L) and PDGFR- $\beta$ ( $65 \mathrm{nmol} / \mathrm{L})$, at pharmacologically relevant concentrations [70]; sustained inhibition of receptor activation ( $\geq 32 \mathrm{~h}$ for VEGFR-2 in vitro) [70]

Inhibits receptor tyrosine kinase FLT-3 and non-receptor tyrosine kinases Lck, Lyn and Src ( $\mathrm{IC}_{50}$ values $\left.16-195 \mathrm{nmol} / \mathrm{L}\right)$ [70], although contribution of inhibition of these kinases to therapeutic activity of nintedanib in ILD is unknown [51]

Inhibited growth factor-induced proliferation of cells (including lung fibroblasts from pts with IPF [71]) in vitro [70, 71]; inhibited growth factor-stimulated fibroblast motility [72] and TGF- $\beta$-stimulated fibroblast to myofibroblast differentiation [73] in lung fibroblasts from pts with IPF; enhanced pro-MMP-2 activity and MMP-2 secretion in lung fibroblasts [71]; reduced TIMP-2 secretion and TGF- $\beta$-induced collagen secretion and deposition in lung fibroblasts [71]

Antifibrotic effects in animal models of pulmonary fibrosis [73], rheumatoid arthritis-associated ILD [74] and systemic sclerosis [75, 76]; anti-inflammatory effects in mouse models of pulmonary fibrosis [73]

No clinically relevant corrected QT interval prolongation when administered at supratherapeutic dosage (200 mg twice daily) for 15 days [51, 77]

\section{Pharmacokinetic properties}

Highly bound to plasma proteins $(\approx 98 \%)$, with albumin being the major binding protein; absolute bioavailability $\approx 5 \%$ following oral administration $(100$ $\mathrm{mg}$ capsule) relative to intravenous in healthy volunteers $[37,51,78]$

$\mathrm{C}_{\text {max }}$ achieved $\approx 2-4 \mathrm{~h}$ after administration of soft capsules under fed conditions; AUC increased by $\approx 20 \%$ and absorption was delayed by $\approx 2 \mathrm{~h}$ after administration in fed vs fasted state (nintedanib should be administered with food to improve gastrointestinal tolerability) $[37,51,78]$

Steady-state plasma concentrations achieved within 1 week and accumulation 1.76-fold (based on AUC) [51]

Predominantly metabolized by hydrolytic ester cleavage resulting in free acid moiety BIBF 1202, which undergoes glucuronidation by UGT1A1,

UGT1A7, UGT1A8 and UGT1A10 to form BIBF 1202 glucuronide; metabolism via CYP pathways is negligible [37, 51, 78]

Effective half-life $9.5 \mathrm{~h}$ in pts with IPF; total plasma clearance was $1390 \mathrm{~mL} / \mathrm{min}$ and renal clearance $20 \mathrm{~mL} / \mathrm{min}$ [51]

$93.4 \%$ of a $100 \mathrm{mg}$ dose eliminated in faeces and $<1 \%$ in urine ( $>90 \%$ of radioactivity recovered within 4 days) $[37,51,78]$

No dose adjustments required in pts with $\mathrm{CL}_{\mathrm{CR}} 30-90 \mathrm{~mL} / \mathrm{min}$; limited experience in pts with $\mathrm{CL}_{\mathrm{CR}}<30 \mathrm{~mL} / \mathrm{min}$ [37, 51]

Increased exposure in pts with hepatic impairment $[37,51,79]$; nintedanib is not recommended for use in pts with moderate or severe hepatic impairment and a reduced dosage (100 $\mathrm{mg}$ twice daily) is recommended in mild hepatic impairment [37, 51]

\section{Drug interactions}

No clinically relevant pharmacokinetic interactions between nintedanib and pirfenidone [37, 51, 80], but adverse reactions may be additive [37]

Substrate of P-gp and, to a minor degree, CYP3A4 [51]; nintedanib tolerability should be closely monitored in pts receiving concomitant P-gp (USA, EU $[37,51]$ ) and CYP3A4 (USA [51]) inhibitors; in the USA, coadministration of nintedanib with P-gp and CYP3A4 inducers should be avoided [51] and in the EU, alternative concomitant drugs with little or no P-gp induction potential should be considered [37]

VEGFR inhibitor (may increase risk of bleeding; Sect. 3); in the USA, nintedanib recipients on full anticoagulation therapy should be closely monitored for bleeding and their anticoagulation treatment adjusted as necessary [51] and in the EU, pts at known risk of bleeding (e.g. receiving a full dose of anticoagulation therapy) should only be treated with nintedanib if expected benefit outweighs possible risk [37]

$A T P$ adenosine triphosphate, $A U C$ area under the concentration-time curve, $C L_{C R}$ creatinine clearance, $C_{\max }$ maximum plasma concentration, $F G F R$ fibroblast growth factor receptor, $F L T$-3 Fms-like tyrosine kinase-3, $I C_{50}$ half-maximal inhibitory concentration, $I L D$ interstitial lung disease, $I P F$ idiopathic pulmonary fibrosis, $M M P$ matrix metalloproteinase, $P$ - $g p$ P-glycoprotein, $P D G F R$ platelet-derived growth factor receptor, $p t(s)$ patient(s), SSc-ILD systemic sclerosis-associated ILD, TGF- $\beta$ transforming growth factor- $\beta$, TIMP-2 tissue inhibitor of metalloproteinase-2, $V E G F R$ vascular endothelial growth factor receptor

The benefit of nintedanib over placebo for the annual rate of FVC decline was consistently demonstrated across prespecified subgroups based on race $[22,23]$ and other baseline characteristics including age, sex, FVC as a percentage of the predicted value, St. George's Respiratory Questionnaire (SGRQ) total score, smoking status, systemic corticosteroid use and bronchodilator use [22]. In post hoc subgroup analyses, nintedanib improved the annual rate of FVC decline relative to placebo irrespective of $\mathrm{DL}_{\mathrm{CO}}$ or composite physiologic index (CPI) [24], presence of preserved lung function (FVC $>90 \%$ of the predicted value) [25], GAP (gender, age, physiology) stage (I vs II/III) [26], diagnostic criteria fulfilled [27], statin use [28] and anti-acid use [29] at baseline. FVC change from baseline to week 52 was similar between nintedanib recipients who had dose reductions and/or treatment interruptions during the trials and those who did not [30].

Nintedanib significantly prolonged the time to first investigator-reported acute exacerbation in INPULSIS-2 but not in INPULSIS-1 nor the pooled analysis (Table 2) [9]. A prespecified sensitivity analysis of pooled data on the time to the first confirmed or suspected acute exacerbation (per adjudication), however, indicated a significant $(p=0.001)$ benefit of nintedanib over placebo [9]. In post hoc pooled analyses, nintedanib significantly $(p<0.05$ vs placebo) reduced the risk of a first acute exacerbation 


\begin{tabular}{|c|c|c|c|}
\hline Study & $\begin{array}{l}\text { Adjusted annual rate of decline in } \\
\text { FVC (mL/year })^{\mathrm{a}}\end{array}$ & $\begin{array}{l}\text { Adjusted mean change from BL in } \\
\text { SGRQ total score }\end{array}$ & $\begin{array}{l}\geq 1 \text { Investigator-reported } \\
\text { acute exacerbation }(\% \text { of } \mathrm{pts})^{\mathrm{b}}\end{array}$ \\
\hline \multicolumn{4}{|l|}{ INPULSIS-1 } \\
\hline Nintedanib $(n=309)$ & -114.7 & 4.34 & 6.1 \\
\hline Placebo $(n=204)$ & -239.9 & 4.39 & 5.4 \\
\hline Difference vs placebo $(95 \% \mathrm{CI})$ & $125.3(77.7 \text { to } 172.8)^{* *}$ & $-0.05(-2.50$ to 2.40$)$ & \\
\hline Hazard ratio $(95 \% \mathrm{CI})$ & & & 1.15 (0.54 to 2.42$)$ \\
\hline \multicolumn{4}{|l|}{ INPULSIS-2 } \\
\hline Nintedanib $(n=329)$ & -113.6 & 2.80 & 3.6 \\
\hline Placebo $(n=219)$ & -207.3 & 5.48 & 9.6 \\
\hline Difference vs placebo $(95 \% \mathrm{CI})$ & $93.7(44.8 \text { to } 142.7)^{* *}$ & $-2.69(-4.95 \text { to }-0.43)^{*}$ & \\
\hline Hazard ratio $(95 \% \mathrm{CI})$ & & & $0.38(0.19 \text { to } 0.77)^{*}$ \\
\hline \multicolumn{4}{|c|}{ INPULSIS-1 and -2 (prespecified pooled analyses) } \\
\hline Nintedanib $(n=638)$ & -113.6 & 3.53 & 4.9 \\
\hline Placebo $(n=423)$ & -223.5 & 4.96 & 7.6 \\
\hline Difference vs placebo $(95 \% \mathrm{CI})$ & $109.9(75.9 \text { to } 144.0)^{* *}$ & $-1.43(-3.09$ to 0.23$)$ & \\
\hline Hazard ratio $(95 \% \mathrm{CI})$ & & & $0.64(0.39$ to 1.05$)$ \\
\hline
\end{tabular}

$B L$ baseline, FVC forced vital capacity, pts patients, SGRQ St. George's Respiratory Questionnaire (range 0-100 points; higher scores indicate worse health-related quality of life)

${ }^{*} p<0.05, * * p<0.001$ vs placebo

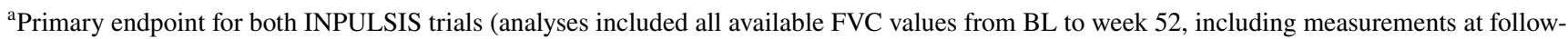
up visits for pts who prematurely discontinued study drug; analyses allowed for missing data, assuming they were missing at random)

${ }^{\mathrm{b}}$ Adjusted mean change from BL in SGRQ total score and time to first investigator-reported acute exacerbation were key secondary endpoints in both INPULSIS trials (assessed over 52-week treatment period and evaluated hierarchically) [fewer pts were included in SQRQ analysis]

reported as a serious $\mathrm{AE}[31]$ and was associated with numerical improvements in 30-, 90- and 180-day mortality following an acute exacerbation [32].

With respect to HR-QOL, nintedanib significantly improved change in SGRQ total score from baseline to week 52 relative to placebo in INPULSIS-2 but not in INPULSIS-1 nor the pooled analysis (Table 2) [9]. Changes in SGRQ domain scores from baseline to week 52 were consistent with those in SGRQ total score [9]. Benefits of nintedanib for HR-QOL may be greater in subgroups with more advanced disease at baseline [33].

Over the 52-week treatment period, the proportion of patients who died from any cause was $5.5 \%$ in the nintedanib group versus $7.8 \%$ in the placebo group [hazard ratio (HR) $0.70 ; 95 \%$ CI 0.43 to 1.12 ] in a prespecified pooled analysis [9]. The rate of death from a respiratory cause was $3.8 \%$ with nintedanib versus $5.0 \%$ with placebo. Between randomization and 28 days after the last dose, deaths occurred in $3.8 \%$ and $6.1 \%$ of nintedanib and placebo recipients [9].

\subsubsection{INPULSIS-ON}

Patients who completed 52 weeks of treatment and the follow-up visit in INPULSIS-1 or INPULSIS- 2 had the opportunity to receive open-label nintedanib in INPULSISON ( $n=430$ previously receiving nintedanib and $n=304$ previously receiving placebo) [10]. During INPULSIS-ON (which ended September 2017), the median exposure to nintedanib was 31.5 months (median total exposure 44.7 months in patients previously receiving nintedanib) and nintedanib was discontinued by $70 \%$ of patients [10].

Slowing of IPF progression was maintained over long-term treatment with nintedanib [10]. During INPULSIS-ON, the mean change in FVC from baseline to week 192 was $-327 \mathrm{~mL}$ in the overall trial population $(n=226$ at week 192$),-349 \mathrm{~mL}$ in patients continuing on nintedanib $(n=138)$ and $-293 \mathrm{~mL}$ in patients who initiated nintedanib therapy in INPULSIS-ON $(n=88)$; the adjusted annual rates of FVC decline calculated over 192 weeks were $-135 \mathrm{~mL} /$ year, $-145 \mathrm{~mL} /$ year and -120 $\mathrm{mL} / \mathrm{year}$ in the corresponding groups. The adjusted annual rate of FVC decline over 192 weeks was generally consistent across subgroups based on age, race or FVC as a percentage of the predicted value at INPULSIS-ON baseline, and was similar irrespective of dose reduction or intensity. The incidences of acute exacerbations were 5.8 and 5.2 per 100 patient-years (PY) in patients who continued nintedanib and patients who initiated nintedanib, respectively. Over $\approx 5$ years' follow-up, $24 \%$ and $27 \%$ of patients in the respective groups died [10]. 


\subsubsection{INSTAGE}

INSTAGE enrolled adults $\geq 40$ years of age diagnosed with IPF within the previous 6 years and with a single-breath $\mathrm{DL}_{\mathrm{CO}}$ (corrected for haemoglobin) $\leq 35 \%$ of the predicted value [12]. Patients received nintedanib $150 \mathrm{mg}$ twice daily plus sildenafil $20 \mathrm{mg}$ three times daily $(n=137)$ or nintedanib $150 \mathrm{mg}$ twice daily plus placebo three times daily $(n=136)$ for 24 weeks. The primary endpoint was change from baseline in SGRQ total score at week 12. At baseline, patients had a mean age of 70 years, FVC $\approx 67 \%$ of the predicted value and $\mathrm{DL}_{\mathrm{co}} \approx 26 \%$ of the predicted value. Mean exposure to nintedanib was $\approx 21$ weeks in each group [12].

The addition of sildenafil to nintedanib did not significantly affect adjusted mean change from baseline in SGRQ total score at week 12 (primary endpoint); results in the nintedanib plus sildenafil arm will not be discussed further [12].

In INSTAGE, nintedanib alone slowed lung function deterioration to a similar rate as that seen in the INPULSIS trials [34]. The adjusted rate of decline in FVC was -66.7 $\mathrm{mL} / 24$ weeks with nintedanib plus placebo in INSTAGE, compared with $-52.3 \mathrm{~mL} / 24$ weeks with nintedanib and $-102.8 \mathrm{~mL} / 24$ weeks with placebo in the INPULSIS trials; the adjusted mean absolute change from baseline in FVC was $-25.5 \mathrm{~mL}$ (vs -25.4 and $-78.8 \mathrm{~mL}$ ) at week 12 and $-58.2 \mathrm{~mL}$ (vs -52.8 and $-106.4 \mathrm{~mL}$ ) at week 24 [34]. In INSTAGE, mean changes in FVC from baseline to week 24 with nintedanib alone were similar between patients with and without emphysema (-54.9 and $-58.2 \mathrm{~mL}$ ) [35].

\subsubsection{Real-World Studies}

Real-world data on the long-term effectiveness of nintedanib in the treatment of IPF are available from a retrospective analysis of data from the European MultiPartner IPF (EMPIRE) registry, which compared nintedanib $(n=637)$, pirfenidone $(n=821)$, switched treatment (i.e. from nintedanib to pirfenidone or vice versa; $n=148$ ) and other or no treatment $(n=1139)$ [19]. The clinical effectiveness of nintedanib $(n=593)$ and pirfenidone $(n=662)$ was also evaluated in a retrospective cohort analysis using data from a US insurance database, in which patients with IPF treated with an antifibrotic drug were one-to-one propensity score matched with untreated patients [17]. In addition, nintedanib treatment was associated with reduced lung function decline in patients with IPF in a prospective study in South Korea ( $n=108)$ [20] and retrospective studies $(n=62-244)$ conducted in Germany [14, 15], Greece [13], Italy [16] and Japan [18]. These data are supported by several smaller studies $(n<60)$ which are not discussed.

Treatment with nintedanib was associated with improved overall survival (OS) and progression-free survival (PFS) in the EMPIRE registry [19]. Median OS from the entry to the registry was 84.7 months with nintedanib, 56.7 months with pirfenidone, 76.9 months with switched treatment and 37.5 months with other/no treatment. At 5 years, the survival rate was 0.62 with nintedanib, 0.46 with pirfenidone, 0.65 with switched treatment and 0.33 with other/no treatment; at 10 years, the survival rates were $0.36,0.22,0.49$ and 0.14 in the respective groups. Median PFS from the entry to the registry was 22.9 months with nintedanib, 20.5 months with pirfenidone, 30.4 months with switched treatment and 17.3 months with other/no treatment [19].

Antifibrotic treatment with nintedanib or pirfenidone was associated with reduced early, but not long-term, mortality in the US insurance database study (mean duration of treatment 229 days with nintedanib and 241 days with pirfenidone) [17]. Patients treated with nintedanib or pirfenidone had significantly reduced all-cause mortality relative to the untreated matched cohort (13.78 vs 16.34 per 100 person-years; $p=0.034$ ) [primary outcome], although the mortality benefit had dissipated by month 24 . There were also significantly fewer acute hospitalizations in the treated cohort (46.70 vs 62.44 per 100 person-years in the untreated cohort; $p<0.001)$. In a subgroup analysis based on antifibrotic treatment, there was no significant difference between nintedanib and pirfenidone recipients in all-cause mortality (14.77 vs 12.83 per 100 person-years) [17].

\subsection{In Other Chronic Fibrosing ILDs with a Progressive Phenotype}

The efficacy of nintedanib in the treatment of patients with progressive fibrosing ILDs was evaluated in the randomized, double-blind, placebo-controlled, multinational, phase III INBUILD trial [36]. This trial enrolled adults with a physician-diagnosed fibrosing ILD, with fibrotic features affecting $>10 \%$ of lung volume on HRCT (as assessed by central review) and fulfilling at least one criteria for ILD progression within the 24 months prior to screening (i.e. relative FVC decline of $\geq 10 \%$ of the predicted value; relative FVC decline of 5 to $<10 \%$ of the predicted value plus worsening respiratory symptoms or an increased extent of fibrosis on HRCT; or worsening respiratory symptoms accompanied by an increased extent of fibrosis) despite receiving standard treatment. An FVC $\geq 45 \%$ of the predicted value and a $\mathrm{DL}_{\mathrm{CO}}$ (corrected for haemoglobin) 30 to $<80 \%$ of the predicted value were required at enrolment [36].

Patients received oral nintedanib $150 \mathrm{mg}$ twice daily or matching placebo [36]. Randomization was stratified by imaging pattern [usual interstitial pneumonia (UIP)-like fibrotic pattern vs other fibrotic patterns], as assessed via central review. Treatment interruptions and/or a dosage reduction to nintedanib $100 \mathrm{mg}$ twice daily were permitted in patients who had AEs. INBUILD Part A encompassed the 
first 52 weeks of the trial; Part B was a variable treatment period beyond week 52 during which patients continued to receive their blinded, randomized treatment until all other patients had completed the trial. The primary endpoint was the annual rate of decline in FVC, assessed over 52 weeks. There were two primary populations: the overall population and patients with a UIP-like fibrotic pattern on HRCT [36].

Baseline characteristics were similar in the two treatment groups and primary populations [36]. In the overall population, patients had a mean age of 66 years, a FVC $69 \%$ of the predicted value and a $\mathrm{DL}_{\mathrm{CO}} 46 \%$ of the predicted value. Chronic hypersensitivity pneumonitis (26\%) and autoimmune ILDs (26\%) were the most common ILD diagnoses. In the overall population, $76 \%$ and $85 \%$ of nintedanib and placebo recipients completed 52 weeks of treatment. During these 52 weeks, the mean durations of exposure to nintedanib and placebo were 10.3 months and 11.2 months [36].

Nintedanib significantly $(p<0.001)$ reduced the annual rate of FVC decline relative to placebo in the overall population and in patients with a UIP-like fibrotic pattern (Table 3) [36]. In these populations, the curves of observed decline from baseline in FVC separated by week 12 and continued to diverge. In patients with other fibrotic patterns, the benefit of nintedanib over placebo for annual rate of FVC decline ( $-79.0 \mathrm{~mL} / \mathrm{year}$ vs $-154.2 \mathrm{~mL} / \mathrm{year}$; between-group difference $75.3 \mathrm{~mL}$; 95\% CI 15.5-135.0) was similar to those in the primary populations [36]. A relative decline from baseline in FVC as a percentage of the predicted value of no greater than 5\% was experienced by $47.6 \%$ of nintedanib recipients versus $31.4 \%$ of placebo recipients [odds ratio (OR) 2.01; 95\% CI 1.46 to $2.76]$ in the overall population, while a relative decline of no greater than $10 \%$ was experienced by $59.3 \%$ versus $51.1 \%$ (OR 1.42; 95\% CI 1.04-1.94) [37].

The benefit of nintedanib for FVC decline was largely consistent across prespecified subgroups based on ILD diagnosis, age, sex, race and baseline FVC [38, 39]. With respect to ILD diagnosis, treatment differences in annual rate of FVC decline were $73.1 \mathrm{~mL} /$ year $(95 \% \mathrm{CI}-8.6$ to 154.8 ) in hypersensitivity pneumonitis, $104.0 \mathrm{~mL} / \mathrm{year}$ (95\% CI 21.1-186.9) in autoimmune ILDs, $141.6 \mathrm{~mL} /$ year (95\% CI 46.0-237.2) in idiopathic non-specific interstitial pneumonia, $68.3 \mathrm{~mL} /$ year $(95 \% \mathrm{CI}-31.4$ to 168.1$)$ in unclassifiable idiopathic interstitial pneumonia, and $197.1 \mathrm{~mL} /$ year (95\% CI 77.6-316.7) in other ILDs [39].

The adjusted mean absolute change from baseline in total King's Brief Interstitial Lung Disease (K-BILD) total score did not meaningfully differ between nintedanib and placebo recipients in the overall population nor in patients with a UIP-like fibrotic pattern (Table 3) [36]. Relative to placebo, nintedanib was associated with less deterioration $(p<0.05)$ from baseline to week 52 in Pulmonary Fibrosis Impact on Quality of Life Scale summary score and Living with Pulmonary Fibrosis dyspnoea and cough symptom domain scores, symptoms score, impacts score and total score [40].

Over the 52-week treatment period, there were no substantial differences in risk of an acute exacerbation of ILD or death, or risk of death alone, between nintedanib and placebo recipients (Table 3) [36]. Over the whole trial

Table 3 Efficacy of nintedanib in the treatment of adults with progressive fibrosing ILDs in the phase III INBUILD trial [36]

\begin{tabular}{|c|c|c|c|c|}
\hline Primary population & 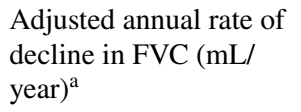 & $\begin{array}{l}\text { Absolute change from BL } \\
\text { in K-BILD total score at } \\
\text { week } 52^{\mathrm{b}}\end{array}$ & $\begin{array}{l}\text { Acute exacerbation of ILD } \\
\text { or death at week } 52 \text { ( } \% \text { of } \\
\text { pts) }\end{array}$ & $\begin{array}{l}\text { Death at week } 52 \\
(\% \text { of pts })^{\mathrm{b}}\end{array}$ \\
\hline \multicolumn{5}{|l|}{ Overall population } \\
\hline Nintedanib $(n=332)$ & -80.8 & 0.55 & 7.8 & 4.8 \\
\hline Placebo $(n=331)$ & -187.8 & -0.79 & 9.7 & 5.1 \\
\hline Difference vs placebo $(95 \% \mathrm{CI})$ & $107.0(65.4 \text { to } 148.5)^{*}$ & $1.34(-0.31$ to 2.98$)$ & & \\
\hline Hazard ratio $(95 \% \mathrm{CI})$ & & & $0.80(0.48$ to 1.34$)$ & $0.94(0.47$ to 1.86$)$ \\
\hline \multicolumn{5}{|c|}{ Pts with a UIP-like fibrotic pattern } \\
\hline Nintedanib $(n=206)$ & -82.9 & 0.75 & 8.3 & 5.3 \\
\hline Placebo $(n=206)$ & -211.1 & -0.78 & 12.1 & 7.8 \\
\hline Difference vs placebo $(95 \% \mathrm{CI})$ & $128.2(70.8 \text { to } 185.6)^{*}$ & $1.53(-0.68$ to 3.74$)$ & & \\
\hline Hazard ratio $(95 \% \mathrm{CI})$ & & & $0.67(0.36$ to 1.24$)$ & $0.68(0.32$ to 1.47$)$ \\
\hline
\end{tabular}

$B L$ baseline, $F V C$ forced vital capacity, $I L D(s)$ interstitial lung disease(s), $K$-BILD King's Brief Interstitial Lung Disease questionnaire (range $0-100$; higher scores represent better health status), pt(s) patient(s), UIP usual interstitial pneumonia

$* p<0.001$ vs placebo

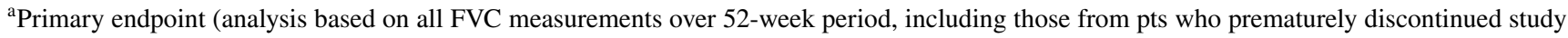
drug; analysis allowed for missing data with assumption they were missing at random)

${ }^{\mathrm{b}}$ Absolute change from BL in K-BILD total score at week 52, time to first acute exacerbation of ILD or death over 52-week period and time to death over 52-week period were main secondary endpoints (not adjusted for multiple comparisons); pt numbers analysed for K-BILD differ 
(mean duration of exposure 15.6 and 16.8 months in the nintedanib and placebo groups), an acute exacerbation of ILD or death occurred in $13.9 \%$ of nintedanib recipients versus $19.6 \%$ of placebo recipients (HR $0.67 ; 95 \%$ CI $0.46-0.98)$ in the overall population and disease progression (i.e. absolute decline in FVC $\geq 10 \%$ of the predicted value) or death occurred in $40.4 \%$ versus $54.7 \%$ (HR 0.66 ; 95\% CI 0.53-0.83); the HR for risk of death was 0.78 (95\% CI 0.50-1.21) [41]. In a subgroup of patients with autoimmune disease-related ILDs $(n=170), 12.2 \%$ of nintedanib recipients versus $20.5 \%$ of placebo recipients died or experienced at least one acute exacerbation of ILD (HR $0.58 ; 95 \%$ CI $0.27-1.27$ ) over the whole trial [42].

\subsection{In SSC-ILD}

The efficacy of nintedanib in the treatment of patients with SSc-ILD was evaluated in the randomized, double-blind, placebo-controlled, multinational, phase III SENSCIS trial [43]. SENSCIS enrolled adults fulfilling the ACR/EULAR classification criteria for systemic sclerosis with onset of first non-Raynaud's symptom within the 7 years prior to screening. SSc-ILD was confirmed by a HRCT scan performed within the 12 months prior to screening showing fibrotic ILD to affect $\geq 10 \%$ of the lungs, as assessed by central review. Patients were also required to have an FVC $\geq 40 \%$ of the predicted value and a $\mathrm{DL}_{\mathrm{CO}}$ (corrected for haemoglobin) $30-89 \%$ of the predicted value. Background therapy with prednisone at a dosage of $\leq 10 \mathrm{mg} / \mathrm{day}$ and/or mycophenolate or methotrexate at a stable dosage for $\geq 6$ months prior to randomization was permitted [43].

Patients received oral nintedanib $150 \mathrm{mg}$ twice daily or matching placebo, with blinded treatment continuing until the last patient reached week 52 (maximum 100 weeks of treatment) [43]. Randomization was stratified based on the presence of antitopoisomerase I antibody (ATA; associated with FVC decline in early systemic sclerosis [44]). The primary endpoint was the annual rate of decline in FVC, assessed over 52 weeks [43].

Baseline characteristics were similar between the treatment arms [43]. Patients had a mean age of 54 years, FVC $73 \%$ of the predicted value and $\mathrm{DL}_{\mathrm{CO}} 53 \%$ of the predicted value. The mean extent of fibrotic ILD (based on assessment of the whole lung) was $36 \%$ and diffuse cutaneous systemic sclerosis was present in $52 \%$ of patients. From the first dose to week 52 , the mean durations of exposure to nintedanib and placebo were 10.5 months and 11.4 months [43].

Nintedanib significantly $(p=0.04)$ reduced the annual rate of FVC decline relative to placebo (Table 4) [43]. Curves for FVC change from baseline separated by week 12 and continued to diverge. At week 52, an absolute decline from baseline in FVC of $>5$ percentage points of the predicted value was experienced by $20.6 \%$ of nintedanib recipients versus $28.5 \%$ of placebo recipients (OR $0.65 ; 95 \%$ CI 0.44-0.96) and an absolute decline of $>10$ percentage points of the predicted value was experienced by $7.0 \%$ versus $8.3 \%$ (OR 0.82; 95\% CI 0.44-1.52) [43].

The benefit of nintedanib over placebo for the annual rate of FVC decline was generally consistent across prespecified subgroups based on age, sex, race, region, ATA status, systemic sclerosis subtype (limited cutaneous vs diffuse cutaneous) and mycophenolate use at baseline $[43,45]$. The adjusted mean annual rate of FVC decline was $-40.2 \mathrm{~mL} /$ year with nintedanib versus $-66.5 \mathrm{~mL} /$ year with placebo in patients using mycophenolate at baseline and $-63.9 \mathrm{~mL} /$ year versus $-119.3 \mathrm{~mL} /$ year in patients not using mycophenolate at baseline [45]. Nintedanib also reduced annual FVC decline relative to placebo irrespective of the extent of lung fibrosis [46] or skin fibrosis [47] at baseline, FVC as a percentage of predicted value at baseline [48] or skin fibrosis progression over 52 weeks [49] (no significant treatment-bytime-by-subgroup interactions).

There were no significant differences between nintedanib and placebo recipients in mean absolute change from baseline in modified Rodnan skin score or SGRQ total score at week 52 (Table 4) [43]. For these key secondary endpoints, results from prespecified subgroup analyses were generally consistent with those in the overall population. Similarly, there were no significant treatment differences for absolute change from baseline in Health Assessment Questionnaire-Disability Index score or Functional Assessment of Chronic Illness Therapy-Dyspnoea score at week 52 [43].

The beneficial effect of nintedanib on lung function deterioration persisted over 100 weeks in analyses of data from the whole SENSCIS trial (median exposure to nintedanib and placebo 15.4 and 15.6 months) [50]. Over 100 weeks, a relative decline in FVC $(\mathrm{mL})$ of $>5 \%$ occurred in $59.4 \%$ of nintedanib recipients versus $69.8 \%$ of placebo recipients (HR $0.80 ; 95 \%$ CI $0.65-0.99 ; p=0.04)$ and a relative decline of $>10 \%$ occurred in $35.8 \%$ versus $40.6 \%$ (HR 0.88 ; $95 \%$ CI $0.67-1.14$ ) [50]. In an exploratory analysis of survival over the whole trial, there was no difference in mortality between nintedanib and placebo recipients $(3.5 \%$ vs $3.1 \%)[37,51]$.

\section{Tolerability of Nintedanib}

Oral nintedanib had a manageable tolerability profile in clinical trials in patients with fibrotic ILDs [9, 11, 12, 36, 52, 53] and in real-world studies in patients with IPF (e.g. [13-16]). In a pooled analysis of safety data from the 52-week INPULSIS- 1 and INPULSIS- 2 trials $(n=638$ and 423 treated with nintedanib and placebo; mean duration of exposure 10.3 months and 10.8 months respectively), the most frequent AEs with nintedanib were diarrhoea, nausea, nasopharyngitis, cough, vomiting, decreased appetite, bronchitis and IPF 
Table 4 Efficacy of nintedanib in the treatment of adults with systemic sclerosis-associated ILD in the phase III SENSCIS trial [43]

Adjusted annual rate of $\quad$ Adjusted mean absolute change decline in FVC (mL/year) $)^{\mathrm{a}}$

from $B L$ in $\mathrm{mRSS}$ at week $52^{\mathrm{b}}$

\begin{tabular}{llll}
\hline Nintedanib $(n=287)$ & -52.4 & -2.17 & 0.81 \\
\hline Placebo $(n=288)$ & -93.3 & -1.96 & -0.88 \\
\hline Difference vs placebo $(95 \% \mathrm{CI})$ & $41.0(2.9 \text { to } 79.0)^{*}$ & $-0.21(-0.94$ to 0.53$)$ & $1.69(-0.73$ to 4.12$)$ \\
\hline
\end{tabular}

$B L$ baseline, $F V C$ forced vital capacity, ILD interstitial lung disease, $p t s$ patients, $m R S S$ modified Rodnan skin score (skin thickness in 17 areas each rated on 0-3 scale; 0 represents normal skin, 3 represents severe thickness with inability to pinch skin into fold), SGRQ St. George's Respiratory Questionnaire (range 0-100 points; higher scores indicate worse health-related quality of life)

${ }^{*} p<0.05$ vs placebo

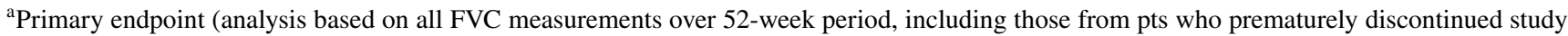
drug; analysis allowed for missing data with assumption they were missing at random)

${ }^{\mathrm{b}}$ Key secondary endpoints (evaluated hierarchically in order displayed); number of pts included in analyses differs from primary analysis

progression (Fig. 1) [54]. Among patients who experienced diarrhoea AEs, these were typically of mild or moderate intensity (in $94.5 \%$ of nintedanib recipients vs $97.4 \%$ of placebo recipients) and resolved without requiring treatment interruption or dose reduction (in $78.6 \%$ vs $98.7 \%$ ). Their first diarrhoea AE typically occurred during the initial 3 months of treatment with nintedanib. Antidiarrhoeal therapies were used by $55.3 \%$ of nintedanib recipients and $25.6 \%$ of placebo recipients who experienced diarrhoea AEs [54].

Drug-related AEs occurred in $71.3 \%$ of nintedanib recipients versus $28.4 \%$ of placebo recipients [54]. AEs were severe in $27.3 \%$ of nintedanib recipients (vs $23.4 \%$ of placebo recipients), serious in $30.4 \%$ (vs $30.0 \%$ ) and fatal in $5.8 \%$ (vs $7.3 \%$ ). AEs led to a permanent dose reduction in a higher proportion of nintedanib than placebo recipients $(15.8 \%$ vs $0.5 \%)$ and led to permanent treatment

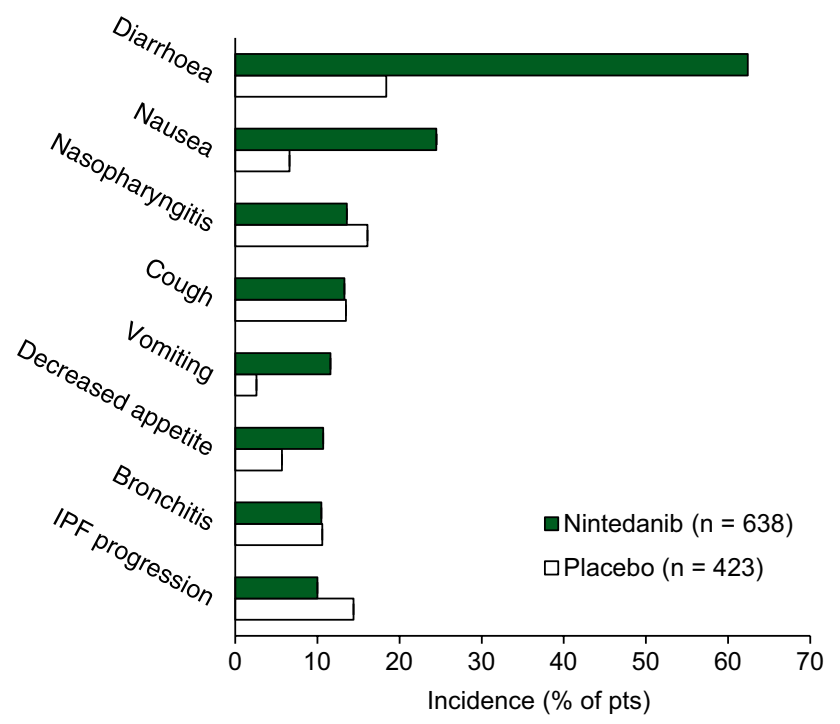

Fig. 1 Most common adverse events with nintedanib (reported in $\geq 10 \%$ of recipients) in the INPULSIS trials (pooled safety data) [54]. IPF idiopathic pulmonary fibrosis discontinuation in $19.3 \%$ of nintedanib recipients versus $13.0 \%$ of placebo recipients. The AEs most frequently leading to discontinuation in nintedanib recipients $(\geq 2 \%)$ were diarrhoea ( $4.4 \%$ vs $0.2 \%$ of placebo recipients), IPF progression $(2.0 \%$ vs $5.0 \%)$ and nausea $(2.0 \%$ vs $0.0 \%)$ [54].

Longer-term safety data from INPULSIS-ON was consistent with that from INPULSIS-1 and INPULSIS-2, revealing no new safety signals [10]. The most frequent AE was diarrhoea, which occurred at a rate of 60.1 events/100 PY in patients who continued on nintedanib and 71.2 events $/ 100$ PY in patients who initiated nintedanib in INPULSIS$\mathrm{ON}$, and led to permanent treatment discontinuation in 5\% (20/430) and $10 \%(31 / 304)$ of patients in the respective groups [10]. Likewise, no new safety signals emerged during the INSTAGE trial in patients with IPF and severe impairment in gas exchange $[12,34]$. In INSTAGE, nintedanib had a similar AE profile to that in the INPULSIS trials in patients with milder disease (albeit with a higher frequency of serious AEs) and AEs led to permanent discontinuation of nintedanib in $19 \%$ of patients who received nintedanib plus placebo [34]. Global pharmacovigilance data for nintedanib in the treatment of IPF collected over 4 years (estimated cumulative exposure to nintedanib of $60,107 \mathrm{PY}$ ) were consistent with the safety profile established in clinical trials [55].

In a 12-week randomized, open-label, phase IV trial (INJOURNEY) in patients with IPF, nintedanib with addon pirfenidone had a manageable safety and tolerability profile in keeping with the AE profile of each drug alone [56] (consistent with results of a phase IV 24-week trial [57]). On-treatment gastrointestinal (GI) AEs occurred in $69.8 \%$ of patients receiving nintedanib plus pirfenidone and $52.9 \%$ receiving nintedanib alone (primary endpoint) [56].

In patients with other progressive fibrosing ILDs (in the INBUILD trial [36]) and SSc-ILD (in the SENSCIS trial $[43,53])$, the AE profile of nintedanib was similar to that in patients with IPF, with GI AEs predominating. 


\subsection{Adverse Events of Special Interest}

GI AEs were the most frequently reported AEs in clinical trials and global pharmacovigilance data. In the latter, diarrhoea had an incidence rate of 301.6 events/1000 PY and most events of diarrhoea were non-serious (97\%) [55]. GI perforation was rare, occurring at a rate of 1.0 event/1000 PY [55]. Diarrhoea should be managed with adequate hydration and antidiarrhoeal medication (e.g. loperamide) and patients with nausea or vomiting should receive appropriate supportive care including anti-emetic therapy if required $[37,51,58]$. If GI AEs cannot be managed with supportive care, dosage modifications or treatment interruptions (or, in severe cases, treatment discontinuation) may be required $[37,51]$.

Drug-induced liver injury, while uncommon, has been associated with nintedanib [37, 51, 58]. In global pharmacovigilance data, elevated liver enzyme or bilirubin levels occurred at a rate of 31.5 events/1000 PY, with a median time to first onset of 60 days [55]. Liver enzyme and bilirubin elevations were typically reversible with dose interruption or reduction in clinical trials [37, 51]. Certain patients may be at higher risk of elevations, including those who are female, Asian or of small physique [37, 51]. Liver function tests should be conducted before initiating treatment and at appropriate intervals thereafter [37, 51, 58]; consult local prescribing information for further details.

VEGF receptor inhibition by nintedanib may increase risk of bleeding (Table 1) [37, 51]. In pooled INPULSIS data, bleeding events were reported in $10.3 \%$ of nintedanib recipients versus $7.8 \%$ of placebo recipients [54]. The incidence of serious bleeding events was similar between treatment groups (1.3\% vs $1.4 \%$ ) [54]. Bleeding occurred at a rate of 36.8 events/1000 PY in global pharmacovigilance data, with the majority of bleeding events being non-serious ( $81 \%$ ) and the most frequently reported type of bleeding event being epistaxis (25\% of events) [55].

Arterial thromboembolic events have been infrequently reported with nintedanib $[37,51]$. In the INPULSIS trials, cardiac disorder AEs occurred in $10.0 \%$ of nintedanib recipients (vs $10.6 \%$ of placebo recipients), serious cardiac disorder AEs occurred in 5.0\% (vs 5.4\%) and fatal cardiac disorders occurred in $0.5 \%$ (vs 1.4\%) [54]. Major cardiovascular AEs and myocardial infarction were reported at rates of 13.4 and 4.3 events/1000 PY in global pharmacovigilance data [55]. Nintedanib use should be approached with caution in patients at higher cardiovascular risk [37, 51]. Treatment interruption should be considered if signs or symptoms of acute myocardial ischaemia develop $[37,51]$.

\section{Dosage and Administration of Nintedanib}

In the EU [37], nintedanib is indicated in adults for the treatment of IPF, other chronic fibrosing ILDs with a progressive phenotype and SSc-ILD. In the USA [51] and Canada [59], nintedanib is indicated for the treatment of IPF, the treatment of chronic fibrosing ILDs with a progressive phenotype and to slow the rate of decline in pulmonary function in patients with SSc-ILD. Nintedanib is available as $150 \mathrm{mg}$ and $100 \mathrm{mg}$ soft capsules [37, 51, 59]. The recommended dosage is 150 mg twice daily administered $\approx 12 \mathrm{~h}$ apart. A reduced dosage of $100 \mathrm{mg}$ twice daily is only recommended for patients who have mild hepatic impairment (Child Pugh A; Table 1) or do not tolerate the higher dose. Capsules should be swallowed whole with water and taken with food (Table 1) [37, 51, 59]. Prior to the initiation of nintedanib, patients should undergo liver function tests and females of reproductive potential should have a pregnancy test $[37,51,59]$. Nintedanib may cause foetal harm when used during pregnancy $[37,51,59]$. In the EU [37] and Canada [59], the use of nintedanib during pregnancy is contraindicated. In the USA, females of reproductive potential should be advised to avoid becoming pregnant while receiving nintedanib [51]. Consult local prescribing information for detailed information regarding missed doses, management of adverse reactions, contraindications, warnings and precautions, and use in special populations.

\section{Place of Nintedanib in the Management of Fibrotic ILDs}

Antifibrotic drugs, while not curative, are capable of slowing ILD progression and have facilitated a paradigm shift in the treatment of IPF over the last decade. In countries such as those of the EU and the USA, the antifibrotics nintedanib and pirfenidone represent the only pharmaceuticals approved for use in IPF (with EU approval of pirfenidone being for mild or moderate disease only [60]). In the most recent international (ATS/ERS/JRS/ALAT) clinical practice guidelines for the treatment of IPF, nintedanib and pirfenidone each received a conditional recommendation for use [61]. Cost concerns may limit their use and must be considered in treatment decisions [61]. Nintedanib is the only drug approved for use in patients with other progressive fibrosing ILDs and SSc-ILD. While nintedanib is not included in the most recent EULAR guidelines for the treatment of systemic sclerosis [62], which were published prior to its approval, a recent European consensus statement names nintedanib as an option for treatment initiation or escalation in patients with SSc-ILD [63]. International treatment guidelines for ILDs other than IPF and SSc-ILD are not available.

Nintedanib was effective in reducing FVC decline, a surrogate endpoint for mortality [64], in the INPULSIS trials in patients with IPF (Sect. 2.1.1); mortality was not considered to be a feasible primary endpoint due to the low mortality rate seen in TOMORROW [65]. The benefit of nintedanib on the annual rate of FVC decline was present across all prespecified subgroups (Sect. 2.1.1) and persisted over an additional 
$\approx 4$ years of treatment in INPULSIS-ON (Sect 2.1.2). Notably, nintedanib appears to reduce FVC decline irrespective of physiological impairment, with the annual rate of FVC decline in nintedanib recipients being comparable between the INSTAGE trial in patients with severe gas exchange impairment and the INPULSIS trials in patients with milder disease (Sect. 2.1.3). Treatment benefits for the risk of acute exacerbation and change in HR-QOL as assessed by SGRQ total score were significant in INPULSIS-2 but not INPULSIS-1 (Sect. 2.1.1). While the difference in mortality did not reach significance in the INPULSIS trials, large real-world studies suggest some improvement in survival with antifibrotic use (Sect. 2.1.4).

Consistent with the overlapping pathophysiology of fibrotic ILDs and the antifibrotic effects of nintedanib across preclinical models of these conditions (Table 1), nintedanib was similarly effective in reducing annual rates of FVC decline in the INBUILD trial in patients with chronic fibrosing ILDs with a progressive phenotype (e.g. chronic hypersensitivity pneumonitis, autoimmune ILDs) [Sect. 2.2] and the SENSCIS trial in patients with SSc-ILD (Sect. 2.3). The benefit of nintedanib on FVC decline was observed irrespective of fibrotic pattern on HRCT in INBUILD and was largely consistent across prespecified subgroups in each trial (Sect. 2). It should be noted that there was no clinical benefit of nintedanib for other manifestations of systemic sclerosis (including skin fibrosis and HR-QOL) in SENSCIS (Sect. 2.3). In INBUILD, changes in K-BILD total score were small in both treatment groups (Sect. 2.2).

Nintedanib had a manageable tolerability profile in patients with IPF in clinical trials and real-world studies (Sect. 3). While GI AEs such as diarrhoea and (to a lesser extent) nausea and vomiting were common with nintedanib, these were mostly of mild to moderate severity. Use of recommended management strategies allowed the majority of patients who experienced GI AEs to continue treatment with nintedanib; $4.4 \%$ of nintedanib recipients discontinued due to diarrhoea AEs during the 52-week INPULSIS trials (Sect. 3). The benefit of nintedanib for FVC decline was maintained in patients who underwent treatment interruptions and/or dose reduction for the management of AEs (Sect. 2.1.1). No new safety signals were identified during INPULSIS-ON or the global pharmacovigilance programme. In patients with other progressive fibrosing ILDs and SSc-ILD, AE profiles were similar to that established in patients with IPF (Sect. 3).

Nintedanib and pirfenidone have distinct mechanisms of action and are thought to target different components of the fibrotic cascade, suggesting the possibility for additive antifibrotic effects [56]. Currently, head-to-head comparisons of nintedanib and pirfenidone in the treatment of IPF are lacking. In the absence of direct comparisons, several network meta-analyses are available and generally indicate no clear differences in efficacy outcomes or rate of serious
AEs between the two drugs (e.g. [66-68]). Both are associated with GI AEs (primarily diarrhoea with nintedanib and nausea with pirfenidone) [69]. Generally, the choice of drug should be reached through joint decision-making between clinician and patient, based on factors such as AE profiles, pill burden (greater with pirfenidone) and tolerability [69].

In conclusion, nintedanib is effective in reducing ILD progression in patients with fibrotic ILDs and has a manageable tolerability profile. Nintedanib continues to represent an important therapeutic option in patients with IPF. Nintedanib is the first drug to be approved for use in other chronic fibrosing ILDs with a progressive phenotype or SScILD, with these approvals expanding the range of fibrotic ILDs for which nintedanib can be prescribed.

\begin{tabular}{|c|c|}
\hline Duplicates removed & 107 \\
\hline $\begin{array}{l}\text { Excluded during initial screening (e.g. press releases; news } \\
\text { reports; not relevant drug/indication; preclinical study; } \\
\text { reviews; case reports; not randomized trial) }\end{array}$ & 47 \\
\hline $\begin{array}{l}\text { Excluded during writing (e.g. reviews; duplicate data; small } \\
\text { patient number; nonrandomized/phase I/II trials) }\end{array}$ & 364 \\
\hline Cited efficacy/tolerability articles & 47 \\
\hline Cited articles not efficacy/tolerability & 33 \\
\hline \multicolumn{2}{|c|}{$\begin{array}{l}\text { Search Strategy: EMBASE, MEDLINE and PubMed from } 1946 \text { to } \\
\text { present. Clinical trial registries/databases and websites were also } \\
\text { searched for relevant data. Key words were nintedanib, Ofev, pulmo- } \\
\text { nary fibrosis, ILD. Records were limited to those in English language. } \\
\text { Searches last updated } 23 \text { Feb } 2021\end{array}$} \\
\hline
\end{tabular}

Acknowledgements During the peer review process, the manufacturer of nintedanib was also offered an opportunity to review this article. Changes resulting from comments received were made on the basis of scientific and editorial merit.

\section{Declarations}

Funding The preparation of this review was not supported by any external funding.

Authorship and Conflict of interest Yvette N. Lamb is a salaried employee of Adis International Ltd/Springer Nature, and declares no relevant conflicts of interest. All authors contributed to the review and are responsible for the article content.

Ethics approval, Consent to participate, Consent to publish, Availability of data and material, Code availability Not applicable.

Open Access This article is licensed under a Creative Commons Attribution-NonCommercial 4.0 International License, which permits any non-commercial use, sharing, adaptation, distribution and reproduction in any medium or format, as long as you give appropriate credit to the original author(s) and the source, provide a link to the Creative Commons licence, and indicate if changes were made. The images or other 
third party material in this article are included in the article's Creative Commons licence, unless indicated otherwise in a credit line to the material. If material is not included in the article's Creative Commons licence and your intended use is not permitted by statutory regulation or exceeds the permitted use, you will need to obtain permission directly from the copyright holder. To view a copy of this licence, visit http://creativecommons.org/licenses/by-nc/4.0/.

\section{References}

1. Brown KK, Martinez FJ, Walsh SLF, et al. The natural history of progressive fibrosing interstitial lung diseases. Eur Respir J. 2020;55:1-10.

2. Cottin V, Wollin L, Fischer A, et al. Fibrosing interstitial lung diseases: knowns and unknowns. Eur Respir Rev. 2019;28:180100.

3. Maher TM, Strek ME. Antifibrotic therapy for idiopathic pulmonary fibrosis: time to treat. Respir Res. 2019;20(205):1-9.

4. Tran T, Šterclová M, Mogulkoc N, et al. The European MultiPartner IPF registry (EMPIRE): validating long-term prognostic factors in idiopathic pulmonary fibrosis. Respir Res. 2020;21:11.

5. Collins BF, Raghu G. Antifibrotic therapy for fibrotic lung disease beyond idiopathic pulmonary fibrosis. Eur Respir Rev. 2019;28:1-15.

6. Cottin V, Hirani NA, Hotchkin DL, et al. Presentation, diagnosis and clinical course of the spectrum of progressive fibrosing interstitial lung diseases. Eur Respir Rev. 2018;27(150):180076.

7. Keating GM. Nintedanib: a review of its use in patients with idiopathic pulmonary fibrosis. Drugs. 2015;75(10):1131-40.

8. McCormack PL. Nintedanib: first global approval. Drugs. 2015;75(1):129-39.

9. Richeldi L, du Bois RM, Raghu G, et al. Efficacy and safety of nintedanib in idiopathic pulmonary fibrosis. N Engl J Med. 2014;370(22):2071-82.

10. Crestani B, Huggins JT, Kaye M, et al. Long-term safety and tolerability of nintedanib in patients with idiopathic pulmonary fibrosis: results from the open-label extension study, INPULSISON. Lancet Respir Med. 2019;7(1):60-8.

11. Richeldi L, Costabel U, Selman M, et al. Efficacy of a tyrosine kinase inhibitor in idiopathic pulmonary fibrosis. N Engl J Med. 2011;365(12):1079-87.

12. Kolb M, Raghu G, Wells AU, et al. Nintedanib plus sildenafil in patients with idiopathic pulmonary fibrosis. N Engl J Med. 2018;379(18):1722-31.

13. Antoniou K, Markopoulou K, Tzouvelekis A, et al. Efficacy and safety of nintedanib in a Greek multicentre idiopathic pulmonary fibrosis registry: a retrospective, observational, cohort study. ERJ Open Res. 2020;6:1-9.

14. Bonella F, Kreuter M, Hagmeyer L, et al. Insights from the German compassionate use program of nintedanib for the treatment of idiopathic pulmonary fibrosis. Respiration. 2016;92(2):98-106.

15. Brunnemer E, Wälscher J, Tenenbaum S, et al. Real-world experience with nintedanib in patients with idiopathic pulmonary fibrosis. Respiration. 2018;95(5):301-9.

16. Cameli P, Refini RM, Bergantini L, et al. Long-term follow-up of patients with idiopathic pulmonary fibrosis treated with pirfenidone or nintedanib: a real-life comparison study. Front Mol Biosci. 2020;7:581828.

17. Dempsey TM, Sangaralingham LR, Yao X, et al. Clinical effectiveness of antifibrotic medications for idiopathic pulmonary fibrosis. Am J Respir Crit Care Med. 2019;200(2):168-74.

18. Dobashi M, Tanaka H, Taima K, et al. The effect of nintedanib on clinical outcome of 158 patients with idiopathic pulmonary fibrosis in real world settings [abstract no. A4766]. Am J Respir Crit Care Med. 2019;199:9.
19. Vasakova M, Sterclova M, Mogulkoc N, et al. Long-term overall survival and progression-free survival in idiopathic pulmonary fibrosis treated by pirfenidone or nintedanib or their switch. Real world data from the EMPIRE registry [abstract no. PA4720]. Eur Respir J. 2019;54:63.

20. Yoon HY, Park S, Kim DS, et al. Efficacy and safety of nintedanib in advanced idiopathic pulmonary fibrosis. Respir Res. 2018;19(203):1-11.

21. Flaherty KR, Kolb M, Vancheri C, et al. Stability or improvement in forced vital capacity with nintedanib in patients with idiopathic pulmonary fibrosis. Eur Respir J. 2018;52:1-8.

22. Costabel U, Inoue Y, Richeldi L, et al. Efficacy of nintedanib in idiopathic pulmonary fibrosis across prespecified subgroups in INPULSIS. Am J Respir Crit Care Med. 2016;193(2):178-85.

23. Taniguchi H, Xu Z, Azuma A, et al. Subgroup analysis of Asian patients in the INPULSIS $®$ trials of nintedanib in idiopathic pulmonary fibrosis. Respirology. 2016;21(8):1425-30.

24. Brown KK, Flaherty KR, Cottin V, et al. Lung function outcomes in the INPULSIS ${ }^{\circledR}$ trials of nintedanib in idiopathic pulmonary fibrosis. Respir Med. 2019;146:42-8.

25. Kolb M, Richeldi L, Behr J, et al. Nintedanib in patients with idiopathic pulmonary fibrosis and preserved lung volume. Thorax. 2017;72(4):340-6.

26. Ryerson CJ, Kolb M, Richeldi L, et al. Effects of nintedanib in patients with idiopathic pulmonary fibrosis by GAP stage. ERJ Open Res. 2019;5:1-9.

27. Raghu G, Wells AU, Nicholson AG, et al. Effect of nintedanib in subgroups of idiopathic pulmonary fibrosis by diagnostic criteria. Am J Respir Crit Care Med. 2017;195(1):78-85.

28. Kreuter M, Costabel U, Richeldi L, et al. Statin therapy and outcomes in trials of nintedanib in idiopathic pulmonary fibrosis. Respiration. 2018;95(5):317-26.

29. Costabel U, Behr J, Crestani B, et al. Anti-acid therapy in idiopathic pulmonary fibrosis: insights from the INPULSIS trials. Respir Res. 2018;19(167):1-9.

30. Koschel D, Maher TM, Inoue Y, et al. Effect of dose reductions and/or interruptions on the efficacy of nintedanib in patients with idiopathic pulmonary fibrosis (IPF): subgroup analysis of the INPULSIS trials [abstract]. Pneumologie. 2018;72(Suppl. 1):S45.

31. Kreuter M, Koegler H, Trampisch M, et al. Differing severities of acute exacerbations of idiopathic pulmonary fibrosis (IPF): insights from the INPULSIS ${ }^{\circ}$ trials. Respir Res. 2019;20(71):1-7.

32. Collard HR, Richeldi L, Kim DS, et al. Acute exacerbations in the INPULSIS trials of nintedanib in idiopathic pulmonary fibrosis. Eur Respir J. 2017;49:1-7.

33. Kreuter M, Wuyts WA, Wijsenbeek M, et al. Health-related quality of life and symptoms in patients with IPF treated with nintedanib: analyses of patient-reported outcomes from the INPULSIS ${ }^{\circledR}$ trials. Respir Res. 2020;21(36):1-12.

34. Richeldi L, Kolb M, Jouneau S, et al. Efficacy and safety of nintedanib in patients with advanced idiopathic pulmonary fibrosis. BMC Pulm Med. 2020;20(3):1-8.

35. Cottin V, Kolb M, Prasse A, et al. Nintedanib plus sildenafil in patients with IPF and severely impaired gas exchange: subgroup analysis by presence of emphysema [abstract no. A4082]. Am J Respir Crit Care Med. 2019;199:9.

36. Flaherty KR, Wells AU, Cottin V, et al. Nintedanib in progressive fibrosing interstitial lung diseases. N Engl J Med. 2019;381(18):1718-27.

37. Boehringer Ingelheim. Ofev (nintedanib): summary of product characteristics. 2020. https://ec.europa.eu/. Accessed 23 Feb 2021.

38. Kolb M, Flaherty KR, Silva R, et al. Effect of nintedanib in patients with progressive fibrosing interstitial lung diseases: subgroup analyses from the INBUILD trial [abstract]. Am J Respir Crit Care Med. 2020;201:A4555. 
39. Wells AU, Flaherty KR, Brown KK, et al. Nintedanib in patients with progressive fibrosing interstitial lung diseases-subgroup analyses by interstitial lung disease diagnosis in the INBUILD trial: a randomised, double-blind, placebo-controlled, parallel-group trial. Lancet Respir Med. 2020;8(5):453-60.

40. Swigris J, Richeldi L, Wijsenbeek M, et al. Effects of nintedanib on dyspnea, cough and quality of life in patients with progressive fibrosing interstitial lung diseases: findings from the INBUILD trial [abstract]. Am J Respir Crit Care Med. 2020;201:A2754.

41. Flaherty KR, Wells AU, Cottin V, et al. Effects of nintedanib on progression of ILD in patients with fibrosing ILDs and a progressive phenotype: further analyses of the INBUILD trial [abstract]. Eur Respir J. 2020;56(Suppl 64):4578.

42. Matteson E, Kell C, Distler A, et al. Effect of nintedanib on progression of interstitial lung disease (ILD) in patients with autoimmune disease-related ILDs: further data from the INBUILD trial [abstract no. OP0115]. Ann Rheum Dis. 2020;76(Suppl 1):76.

43. Distler O, Highland KB, Gahlemann M, et al. Nintedanib for systemic sclerosis-associated interstitial lung disease. N Engl J Med. 2019;380(26):2518-28.

44. Assassi S, Sharif R, Lasky RE, et al. Predictors of interstitial lung disease in early systemic sclerosis: a prospective longitudinal study of the GENISOS cohort. Arthritis Res Ther. 2010;12:R166.

45. Highland KB, Distler O, Kuwana M, et al. Efficacy and safety of nintedanib in patients with systemic sclerosis-associated interstitial lung disease treated with mycophenolate: a subgroup analysis of the SENSCIS trial. Lancet Respir Med. 2021;9(1):96-106.

46. Raghu G, Distler O, Azuma A, et al. Effects of nintedanib in patients with systemic sclerosis-associated ILD (SSc-ILD) and differing extents of lung fibrosis: the SENSCIS trial [abstract no. PA5193]. Eur Respir J. 2019;54:63.

47. Allanore Y, Steen V, Kuwana M, et al. Effects of nintedanib in patients with systemic sclerosis-associated ILD (SSc-ILD) and differing extents of skin fibrosis: further analyses of the SENSCIS trial [abstract no. THU0330]. Ann Rheum Dis. 2020;76(Suppl 1):395-6.

48. Maher T, Distler O, Azuma A, et al. Effects of nintedanib in patients with systemic sclerosis-associated ILD (SSc-ILD) and differing FVC at baseline: the SENSCIS trial [abstract no. OA3599]. Eur Respir J. 2019;54:63.

49. Distler O, Highland K, Hoffmann-Vold AM, et al. Correlation between progression of skin fibrosis and progression of interstitial lung disease (ILD) in patients with SSc-ILD: data from the SENSCIS trial [abstract no. SAT0313]. Ann Rheum Dis. 2020;76(Suppl 1):1101-2.

50. Maher T, Distler O, Allanore Y, et al. Effect of nintedanib on progression of systemic sclerosis-associated interstitial lung disease (SSc-ILD) beyond 52 weeks: data from the SENSCIS trial [abstract]. Am J Respir Crit Care Med. 2020;201:A4558.

51. Boehringer Ingelheim. OFEV (nintedanib): US prescribing information. 2020. https://dailymed.nlm.nih.gov/. Accessed 23 Feb 2021.

52. Lancaster L, Crestani B, Hernandez P, et al. Safety and survival data in patients with idiopathic pulmonary fibrosis treated with nintedanib: pooled data from six clinical trials. BMJ Open Respir Res. 2019;6(e000397):1-7.

53. Seibold JR, Maher TM, Highland KB, et al. Safety and tolerability of nintedanib in patients with systemic sclerosis-associated interstitial lung disease: data from the SENSCIS trial. Ann Rheum Dis. 2020;79:1478-84.

54. Corte T, Bonella F, Crestani B, et al. Safety, tolerability and appropriate use of nintedanib in idiopathic pulmonary fibrosis. Respir Res. 2015;16(116):1-10.

55. Lasky JA, Criner GJ, Lazarus HM, et al. Safety of nintedanib in patients with idiopathic pulmonary fibrosis: global pharmacovigilance data. Adv Ther. 2020;37:4209-19.
56. Vancheri C, Kreuter M, Richeldi L, et al. Nintedanib with add-on pirfenidone in idiopathic pulmonary fibrosis. Results of the INJOURNEY trial. Am J Respir Crit Care Med. 2018;197(3):356-63.

57. Flaherty KR, Fell CD, Huggins JT, et al. Safety of nintedanib added to pirfenidone treatment for idiopathic pulmonary fibrosis. Eur Respir J. 2018;52:1-10.

58. Bendstrup E, Wuyts W, Alfaro T, et al. Nintedanib in idiopathic pulmonary fibrosis: practical management recommendations for potential adverse events. Respiration. 2019;97(2):173-84.

59. Boehringer Ingelheim. OFEV ® (nintedanib capsules): product monograph. 2020. https://www.boehringer-ingelheim.ca/. Accessed 23 Feb 2021.

60. NICE. Pirfenidone for treating idiopathic pulmonary fibrosis 2018. https://www.nice.org.uk/. Accessed 23 Feb 2021.

61. Raghu G, Rochwerg B, Zhang Y, et al. An official ATS/ERS/JRS/ ALAT clinical practice guideline: treatment of idiopathic pulmonary fibrosis. Am J Respir Crit Care Med. 2015;192(2):e1-19.

62. Kowal-Bielecka O, Fransen J, Avouac J, et al. Update of EULAR recommendations for the treatment of systemic sclerosis. Ann Rheum Dis. 2017;76:1327-39.

63. Hoffmann-Vold AM, Maher TM, Philpot EE, et al. The identification and management of interstitial lung disease in systemic sclerosis: evidence-based European consensus statements. Lancet Rheumatol. 2020;2(2):e71-83.

64. Ley B. Clarity on endpoints for clinical trials in idiopathic pulmonary fibrosis. Ann Am Thorac Soc. 2017;14(9):1383-4.

65. Richeldi L, Cottin V, Flaherty KR, et al. Design of the INPULSIS $^{\text {TM }}$ trials: two phase 3 trials of nintedanib in patients with idiopathic pulmonary fibrosis. Respir Med. 2014;108(7):1023-30.

66. Canestaro WJ, Forrester SH, Raghu G, et al. Drug treatment of idiopathic pulmonary fibrosis: systematic review and network meta-analysis. Chest. 2016;149(3):756-66.

67. Rochwerg B, Neupane B, Zhang Y, et al. Treatment of idiopathic pulmonary fibrosis: a network meta-analysis. BMC Med. 2016;14:18.

68. Skandamis A, Kani C, Markantonis SL, et al. Systematic review and network meta-analysis of approved medicines for the treatment of idiopathic pulmonary fibrosis. J Drug Assess. 2019;8(1):55-61.

69. Hayton $\mathrm{C}$, Chaudhuri N. Managing idiopathic pulmonary fibrosis: which drug for which patient? Drugs Aging. 2017;34(9):647-53.

70. Hilberg F, Roth GJ, Krssak M, et al. BIBF 1120: triple angiokinase inhibitor with sustained receptor blockade and good antitumor efficacy. Cancer Res. 2008;68(12):4774-82.

71. Hostettler KE, Zhong J, Papakonstantinou E, et al. Anti-fibrotic effects of nintedanib in lung fibroblasts derived from patients with idiopathic pulmonary fibrosis. Respir Res. 2014;15(1):157.

72. Wollin L, Wex E, Pautsch A, et al. Mode of action of nintedanib in the treatment of idiopathic pulmonary fibrosis. Eur Respir J. 2015;45(5):1434-45.

73. Wollin L, Maillet I, Quesniaux V, et al. Antifibrotic and antiinflammatory activity of the tyrosine kinase inhibitor nintedanib in experimental models of lung fibrosis. J Pharmacol Exp Ther. 2014;349(2):209-20.

74. Redente EF, Aguilar MA, Black BP, et al. Nintedanib reduces pulmonary fibrosis in a model of rheumatoid arthritis-associated interstitial lung disease. Am J Physiol Lung Cell Mol Physiol. 2018;314:L998-1009.

75. Huang J, Beyer C, Palumbo-Zerr K, et al. Nintedanib inhibits fibroblast activation and ameliorates fibrosis in preclinical models of systemic sclerosis. Ann Rheum Dis. 2016;75(5):883-90.

76. Huang J, Maier C, Zhang Y, et al. Nintedanib inhibits macrophage activation and ameliorates vascular and fibrotic manifestations in the Fra2 mouse model of systemic sclerosis. Ann Rheum Dis. 2017;76:1941-8. 
77. Eisen T, Shparyk Y, Macleod N, et al. Effect of small angiokinase inhibitor nintedanib (BIBF 1120) on QT interval in patients with previously untreated, advanced renal cell cancer in an open-label, phase II study. Invest New Drugs. 2013;31(5):1283-93.

78. Wind S, Schmid U, Freiwald M, et al. Clinical pharmacokinetics and pharmacodynamics of nintedanib. Clin Pharmacokinet. 2019;58(9):1131-47.
79. Marzin K, Kretschmar G, Luedtke D, et al. Pharmacokinetics of nintedanib in subjects with hepatic impairment. J Clin Pharmacol. 2018;58(3):357-63.

80. Richeldi L, Fletcher S, Adamali H, et al. No relevant pharmacokinetic drug-drug interaction between nintedanib and pirfenidone. Eur Respir J. 2019;53:1. 